\title{
Retour aux origines et aux années 1820 : de l'atelier de charronnage primitif anglais aux premiers dépôts organisés en France par Marc Seguin
}

Back to the origins and the 1820's: from the primitive English workshop of charronnage to the first depots organized in France by Marc Séguin

Clive Lamming

\section{OpenEdition Journals}

Édition électronique

URL : https://journals.openedition.org/rhcf/1774

DOl : 10.4000/rhcf.1774

Éditeur

Rails \& histoire

Édition imprimée

Date de publication : 1 décembre 2003

Pagination : 257-274

ISBN : 0996-9403

ISSN : 0996-9403

Référence électronique

Clive Lamming, «Retour aux origines et aux années 1820 : de l'atelier de charronnage primitif anglais aux premiers dépôts organisés en France par Marc Seguin », Revue d'histoire des chemins de fer [En ligne], 28-29 | 2003, mis en ligne le 30 décembre 2014, consulté le 22 avril 2022. URL : http:// journals.openedition.org/rhcf/1774 ; DOI : https://doi.org/10.4000/rhcf.1774 


\section{Retour aux origines et aux années 1820 : de I'atelier de charronnage primitif anglais aux premiers dépôts organisés en France par Marc Seguin}

Faire l'histoire des techniques, c'est aussi accorder aux commencements toute l'importance qu'ils méritent. Et quand on étudie le passé, on découvre que les commencements fixent pour de très longues périodes des manières de penser, d'agir, et de fabriquer qui peuvent ultérieurement sembler dénués de logique pour l'historien qui « prend le train en marche ». Le chemin de fer français est bien né en Angleterre et, quand Marc Seguin effectue ses voyages en Angleterre pour opérer ce que l'on appellerait aujourd'hui un « transfert de technologie », il trouve un ensemble technique beaucoup plus ancien, riche et complexe qu'on ne le suppose aujourd'hui' ${ }^{1}$.

De nombreuses lectures nous ont donné l'impression que le chemin de fer commence avec la locomotive de Trevithick en 1804, la ligne de Stockton à Darlington en 1825, la Fusée de Stephenson et le concours de Rainhill en 1829. C'est inexact : le commencement n'est pas à chercher seulement dans cette époque pourtant riche et féconde dans la mesure où elle est celle du transfert du chemin de fer en France et sur le continent européen ou américain, mais encore plus loin en avant dans le temps.

Marc Seguin ne visite pas un chemin de fer nouveau, en commencement, mais, plutôt, le chemin de fer qui est en train de tourner la page d'une grande et longue époque. Une époque déjà ancienne, qui a commencé au Xvie siècle, avec le chemin de fer anglais d'avant la locomotive à vapeur, vieux déjà de plus d'un siècle, aux nombreuses lignes disséminées dans l'ensemble du Royaume-Uni.

Marc Seguin : importateur d'un chemin de fer anglais déjà ancien

Marc Seguin ne se voit nullement comme le créateur des fondements techniques des chemins de fer en France. Homme d'action, il veut, au contraire, aller vite et rapporter avec lui ce qui marche déjà.

1 - À propos de Marc Seguin, voir dans la $R H C F$ «Les débuts de la ligne ferroviaire de Saint-Étienne à Lyon et les événements de 1830 », RHCF 26 (print. 2002), p. 218-228 et «Définition de la voie ferrée moderne : la synthèses du Saint-Étienne - Lyon (18251835) ", RHCF 27 (aut. 2002), p. 7-26, par Michel Cotte, auteur de Innovation et transfert de technologies, le cas des entreprises de Marc Seguin (France 1815-1835), thèse, 1995 (NdlR). 
Pour gagner du temps et développer la région de Saint-Étienne autour d'un chemin de fer, il entreprend un voyage en Angleterre. Ce sont bien là les prémices de ce que l'on appellerait aujourd'hui un « transfert de technologie », car Marc Seguin n'est pas à compter parmi les pionniers du chemin de fer : il en est simplement l'importateur en France. Il l'écrit lui-même :

Voué à l'industrie depuis ma jeunesse, je me suis occupé surtout d'améliorer en France le système des communications. Quelques voyages en Angleterre m'avaient convaincu que, pour transporter dans ma patrie la civilisation industrielle de la nation anglaise, il fallait, avant tout, mettre nos moyens de transport à l'unisson des siens... ${ }^{2}$

Dès 1822, Marc Seguin songe à son voyage, d'après une lettre adressée à Augustine, son épouse. Il séjourne à Londres en novembre 1823, puis de l'hiver de 1826 au printemps de 1827. Il rencontre des personnalités scientifiques de l'époque comme Herschel ou Faraday mais, surtout, il s'intéresse aux chantiers de construction de lignes de chemin de fer et fait la connaissance de George Stephenson.

En matière de chemins de fer, Seguin ne découvre pas une époque de pionniers et de tâtonnements, bien au contraire. Le chemin de fer est une chose mûre et établie, formant un moyen de transport industriel reconnu, vieux de plus de 150 ans, et connaissant déjà, depuis plus de trente ans, des essais en traction vapeur. Prenant conscience de l'avance considérable des Anglais dans ce domaine, puisque les rails de bois remontent à 1649 (Newcastle), ceux en fonte à la décennie 1760, puisque les locomotives à vapeur circulent dès 1801, Marc Seguin veut absolument transférer cette avance technologique en France ; il prend des notes, discute avec les ingénieurs, les ouvriers, et visite un grand nombre de lignes alors en service.

\section{Ce que Marc Seguin a vu et aurait pu voir}

Nous savons qu'il se rend sur le chemin de fer de Leeds, de Sunderland (un port près de Newcastle), et de Stockton à Darlington ${ }^{3}$. Il se déplace donc jusque dans le nord de l'Angleterre, ne ménageant pas sa peine et couvrant d'importantes distances.

2- De l'influence des chemins de fer et de l'art de les tracer et de les construire, Lyon, Pitrat Aîné, 1839 (2 éd. 1887), Introduction.

3- D’après Ferdinand Achard, article paru dans The Locomotive Magaqine, numéro du 15 mars 1927. 
Puisque Seguin a séjourné plusieurs fois à Londres, il a pu trouver le temps d'aller visiter le vieux Surrey Iron Railway : nous disons bien «vieux », car ce chemin de fer, ayant bénéficié de la toute première concession publique dans l'histoire mondiale du chemin de fer, date du 21 mai 1801, et accuse donc déjà un âge approchant la trentaine quand Marc Seguin fait son voyage. Ce chemin de fer relie Wandsworth, un port de la Tamise près de Clapham (future bifurcation ferroviaire qui sera très connue pour son trafic record), et aboutit à une usine située à Croydon. La ligne ferma en 1846, faute de performances par manque de traction vapeur, et victime d'un système de rails plats à rebord de guidage situé vers l'intérieur de la voie, solution complètement incompatible avec le réseau qui s'était alors construit.

Il aura pu, aussi y visiter le Sirhony Railway dont le réseau s'étend dans le pays de Galles et il aurait pu, s'il était retourné en 1829, voir une locomotive type 030, la $16^{\mathrm{e}}$ construite par Stephenson et désignée par le constructeur sous le terme de type « $B$ », pesant entre 8 et 9 tonnes en ordre de marche ${ }^{4}$. Ressemblant à la Fusée dont elle est la contemporaine, elle possède deux cylindres situés à l'arrière près de la plate-forme de conduite, actionnant directement le premier des trois essieux accouplés.

Le chemin de fer desservant les mines de Hetton auraient pu mériter une visite : en novembre 1822, ce réseau long de 8 miles adopte la traction à vapeur, avec des locomotives Stephenson. Non seulement Marc Seguin les aurait trouvées en service intensif vers la fin des années 1820, mais d'autres peuvent les voir et les photographier en 1912, toujours actives, comme nous le montrons figure 16.

Peut-être a-t-il visité le Forest of Dean Tramroad, construit à partir de 1807 et toujours en service 80 années plus tard, maintenu dans sa forme primitive et toujours à traction animale, utilisant encore les wagons en bois des débuts. Le réseau a totalisé 180 miles, soit plus de 280 kilomètres lors de son extension maximale, et l'écartement est de 48 pouces, soit 1,22 mètre. Des ouvrages d'art, des remblais et des tranchées ont été construits en grande quantité, préfigurant l'établissement des lignes de chemin de fer modernes, préfigurant aussi les travaux que Marc Seguin fera sur la ligne de Saint-Étienne à Lyon (fig. 13 et 14).

4- D’après The Locomotive Magarine, janvier 1915. 


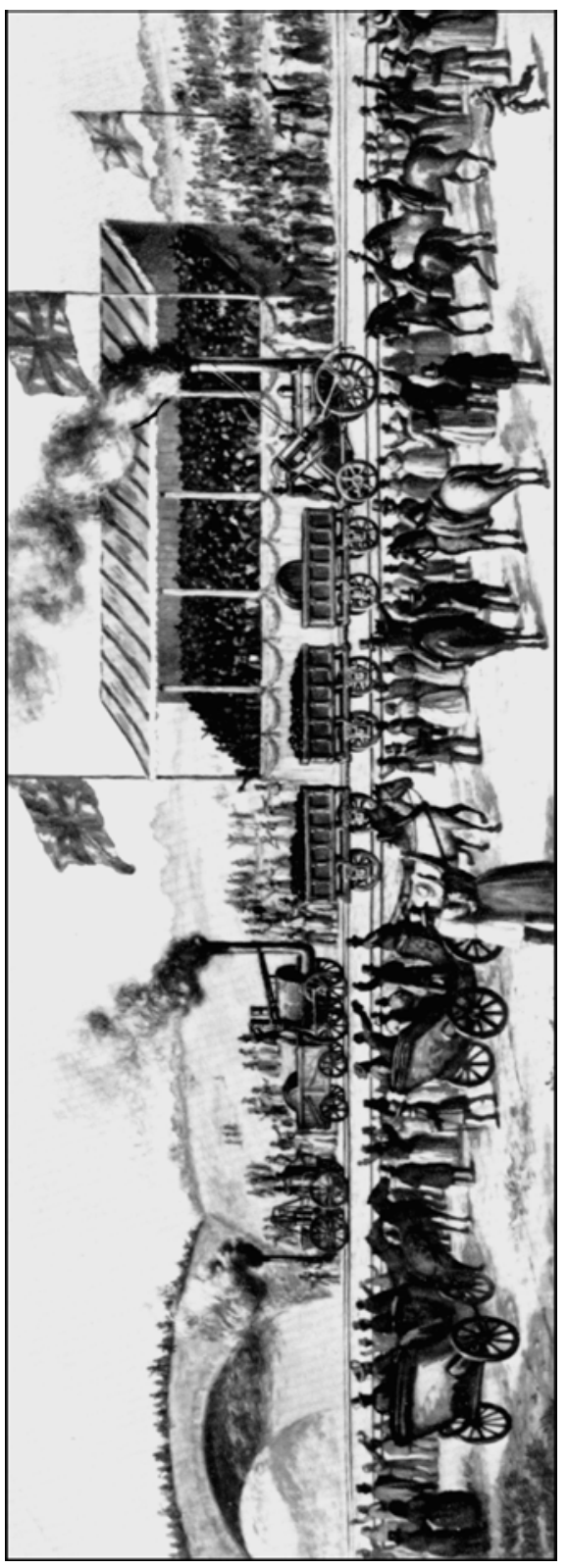

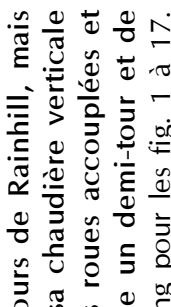

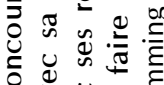

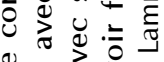
는 䒕苋造

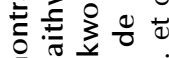

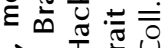
๑ับ㐫 ㄷํㅇ 웜 유웡

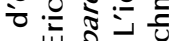
का

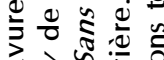

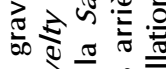

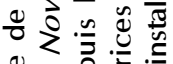
은

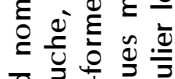
ช

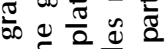

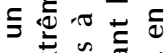
引 区

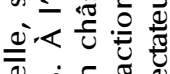
ป

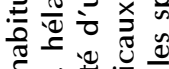

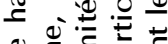
ว

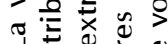

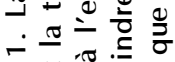
๒

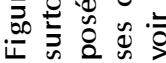



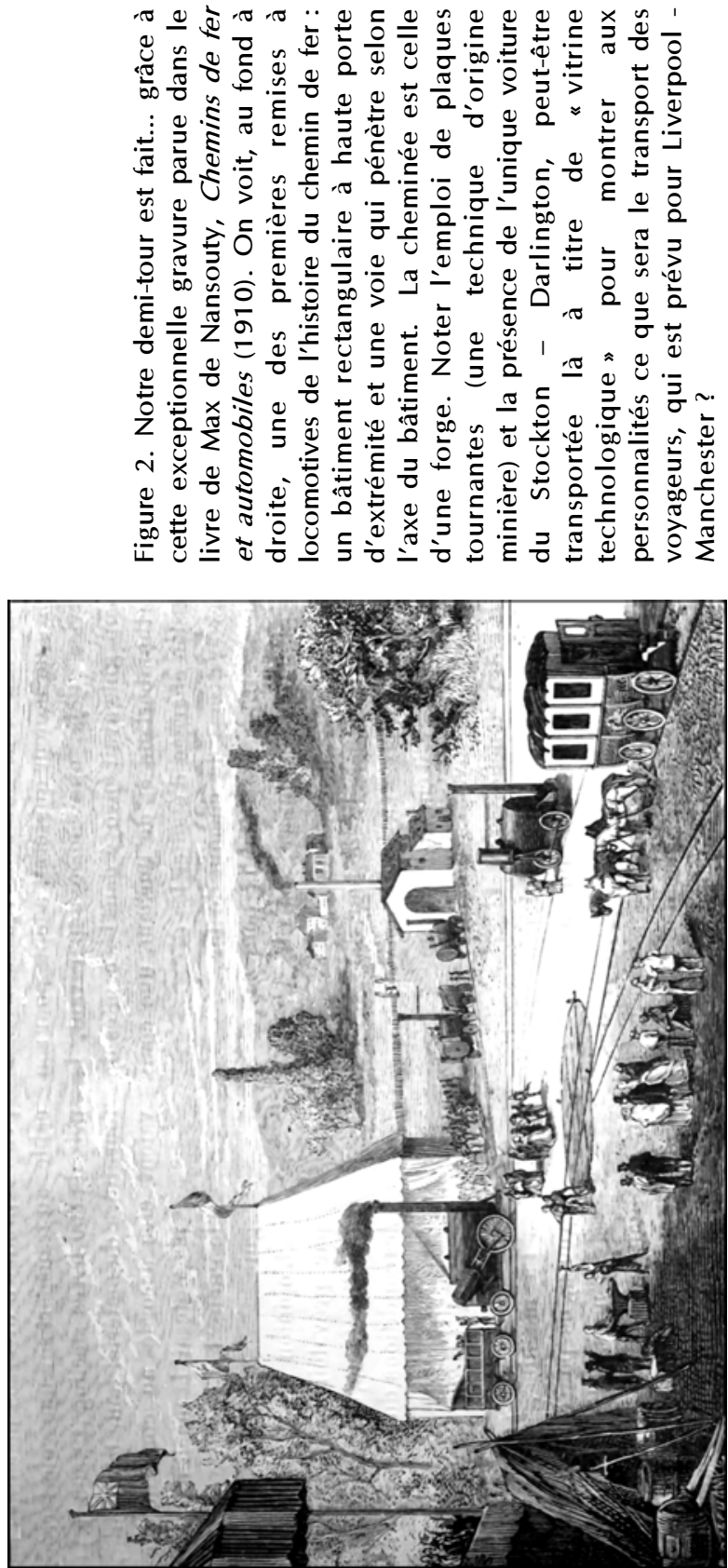
Figure 3.

Approchonsnous de ce b â $t$ i m e n t, grâce à une photographie prise vers 1880 qui montre le

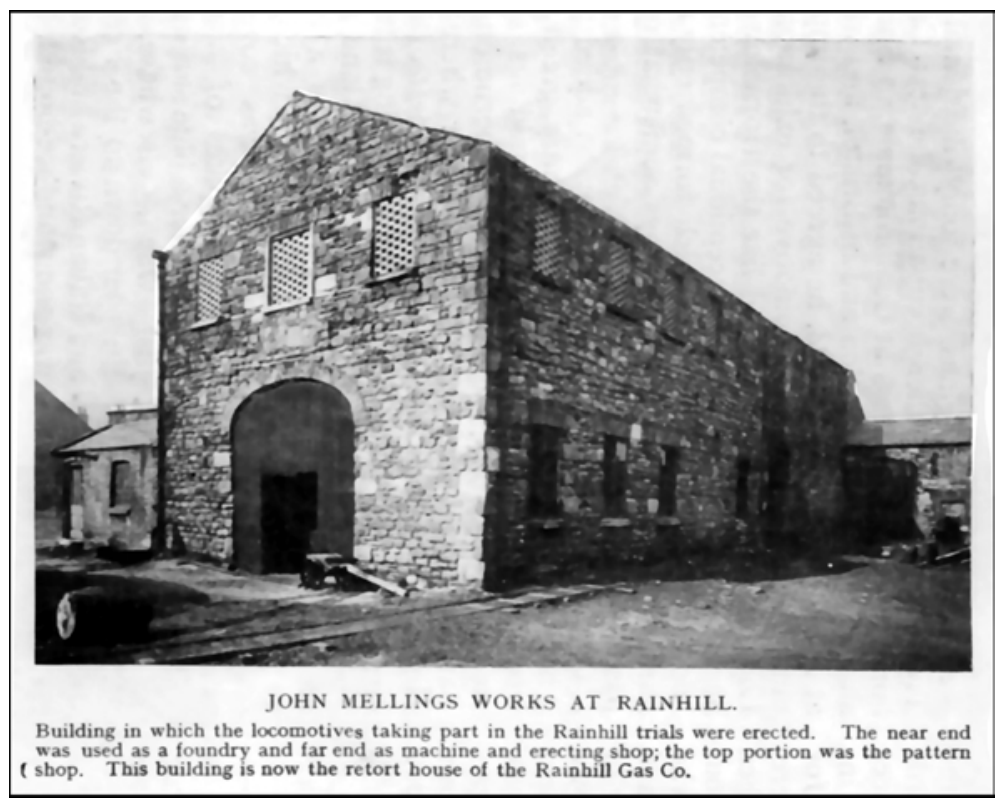
corps de bâtiment d'origine. II a été transformé en usine à gaz, d'où les ouvertures avec treillis de bois, mais la disposition générale du bâtiment est celle des premières "remises - ateliers" du chemin de fer à traction vapeur.

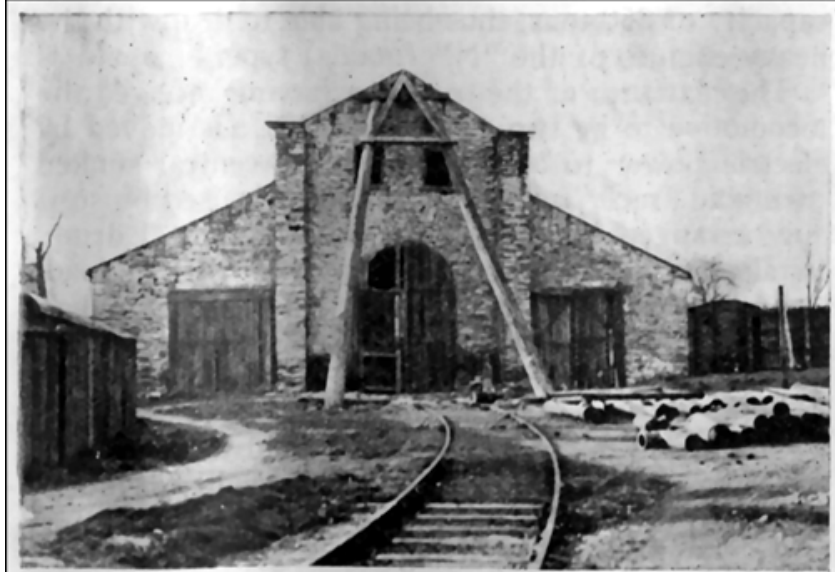

MR. TIMOTHY HACKWORTH'S ERECTING SHOP FOR LOCOMOTIVES, SOHO ENGINE WORKS, SHILDON, 1834. EAST VIEW.
Figure 4. D'autres remisesateliers de ce genre ont été construits en Angleterre, pour les débuts de la locomotive à vapeur. Par exemple, I'atelier de Hackworth, datant de 1834, photographié vers 1890, document paru dans The Locomotive Magazine de 1898. Noter le système en bois destiné au levage des lourdes pièces : il est accolé à l'extérieur du bâtiment. 
Figure 5. Cette vue, prise vers 1860 à Liskeard montre un dépôt dont l'état de vétusté peut le faire remonter aux débuts de la locomotive à vapeur. On retrouve toujours cette disposition longitudinale avec une (ou deux, maintenant) voies pénétrant dans I'axe du bâtiment et, toujours aussi, le système de levage extérieur, placé contre la façade.
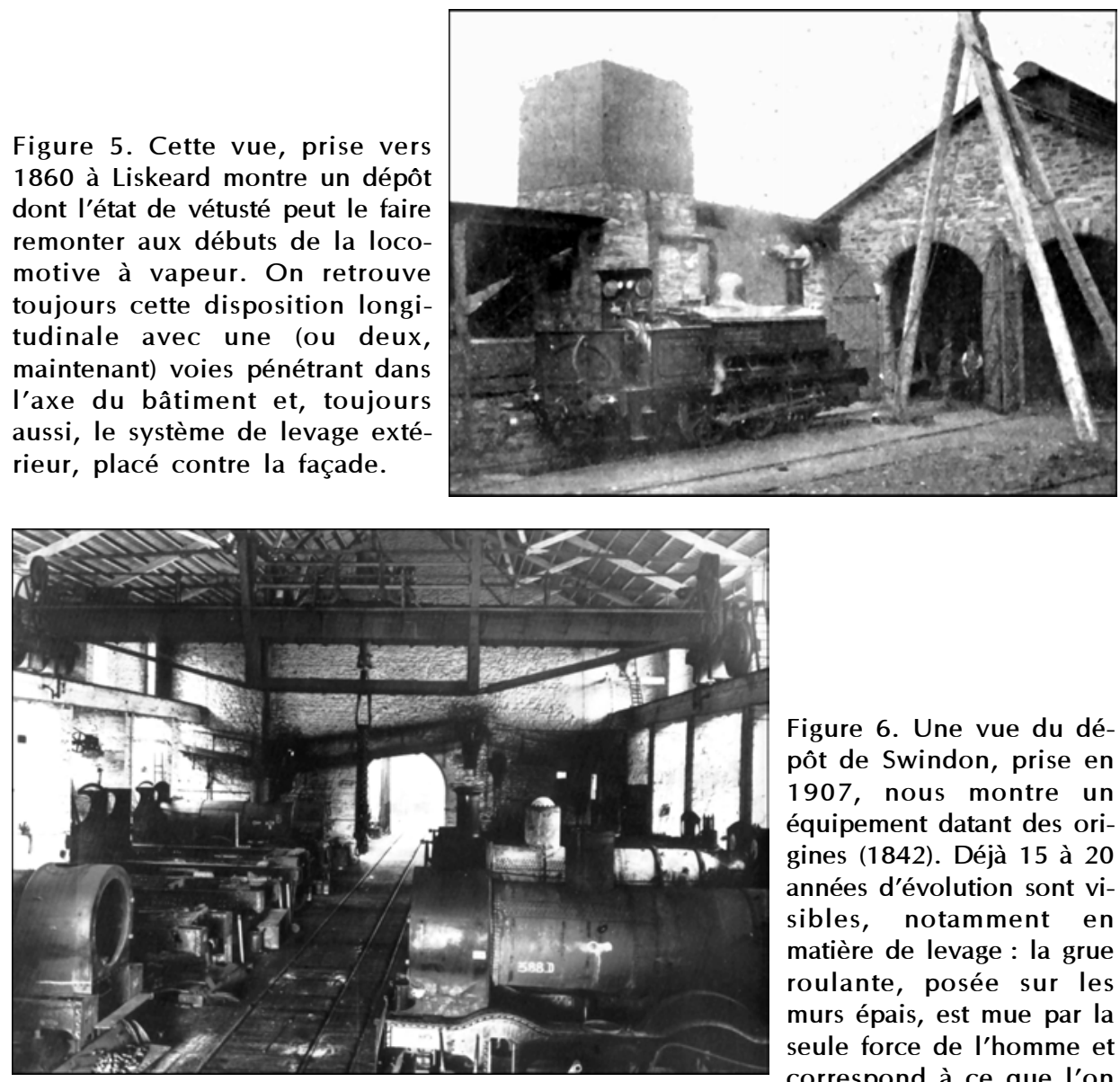

Figure 6. Une vue du dépôt de Swindon, prise en 1907, nous montre un équipement datant des origines (1842). Déjà 15 à 20 années d'évolution sont visibles, notamment en matière de levage : la grue roulante, posée sur les murs épais, est mue par la seule force de I'homme et correspond à ce que I'on trouve dans les ateliers de charronnage du siècle précédent. La disposition des locomotives, placées côte à côte, perpendiculairement à une voie d'accès centrale desservie par un pont transbordeur situé à I'extérieur de I'atelier, est désormais la règle.

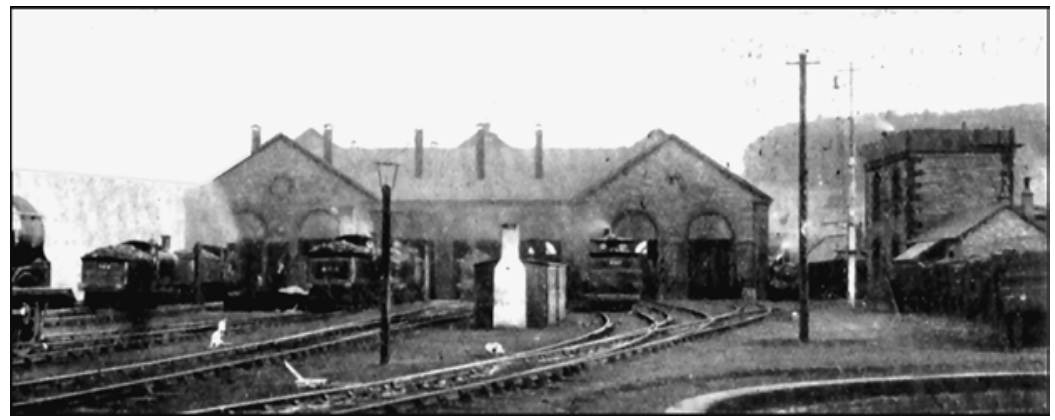

Figure 7. La pratique anglaise des installations sur voies parallèles, sans pont tournant, reste en vi g u e u r j u s q u' à

aujourd'hui. Ici le dépôt de Perth, en Angleterre, vu durant les années 1870. Le bâtiment est du type primitif élargi à 7 voies, avec deux ailes et un corps central en retrait. 


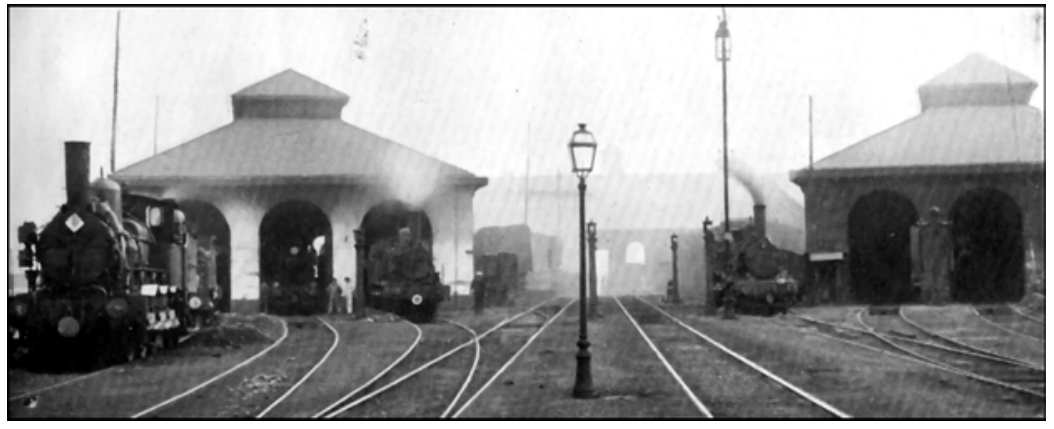

Figures 8 et 9. Deux vues du dépôt de La Chapelle nous montrent, vers 1880, le bâtiment ancien du dépôt. La disposition privilégiée dès les débuts du chemin de fer est la remise à voies parallèles et, pour les grands dépôts, la juxtaposition de plusieurs corps de bâtiments ayant deux ou trois entrées. Sur la deuxième vue, on constate que l'étroitesse des ouvertures comme l'étroitesse générale s'accordent mal avec les dimensions croissantes des locomotives, notamment pour le gabarit en largeur. L'Outrance $\left(1^{\mathrm{er}}\right.$ type, chaudière non reconstruite) permet de dater la photographie des années postérieures à 1877 .

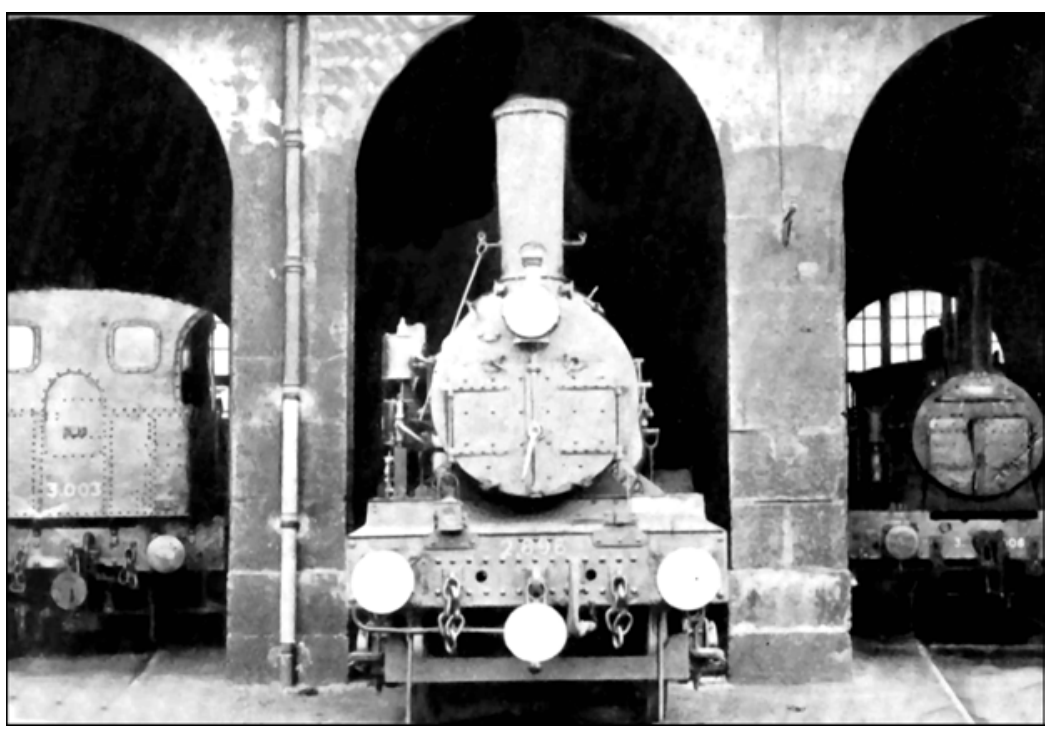




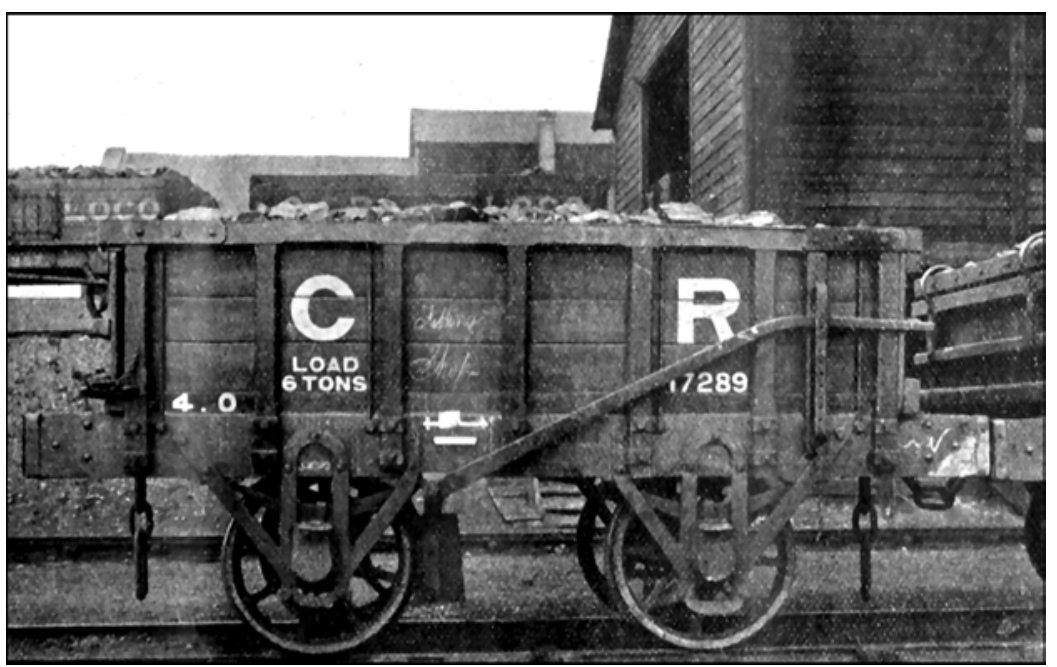

Figures 10 et 11 . Le matériel roulant des années 1830-1840, encore en circulation vers 1870 , témoigne de la conception " charronnage " avec une construction en bois, des pièces de liaison forgées, et laisse clairement apparaître la nature du travail de construction et de réparation effectué dans les ateliers.

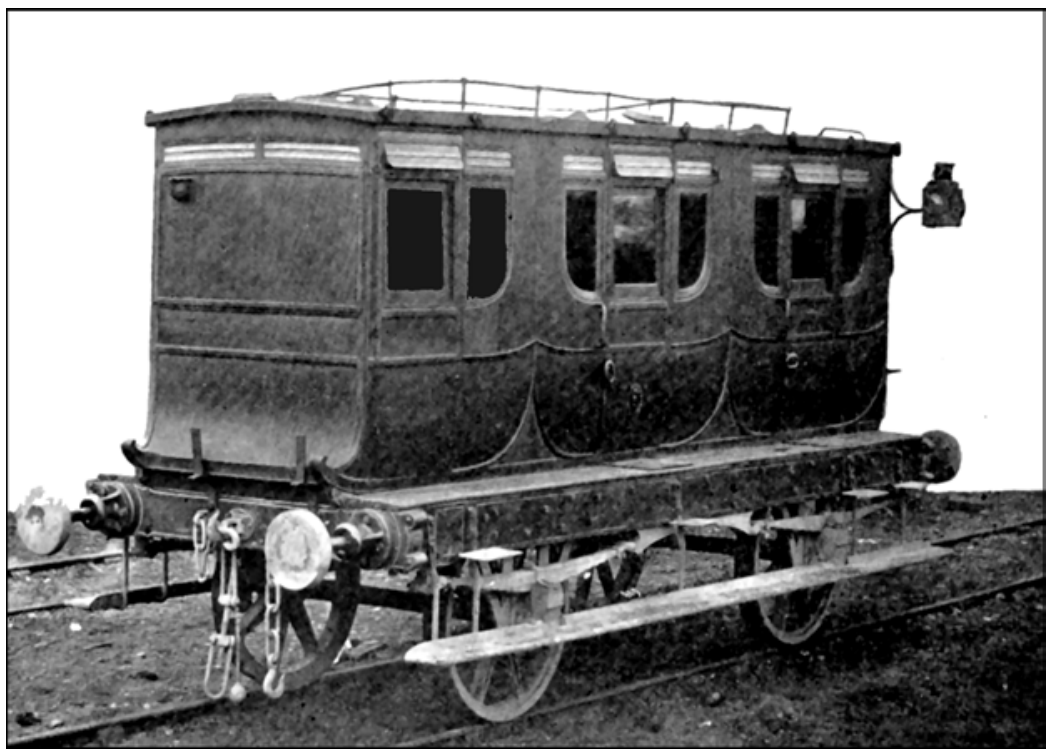




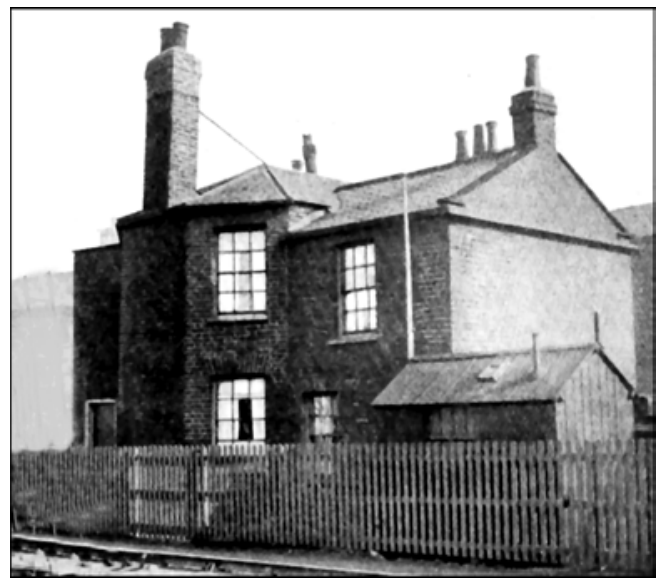

Figure 12. La gare de Stockton construite en 1822. La simplicité des bâtiments est évidente, et les architectes industriels ne sont pas encore intervenus pour construire des gares organisées en fonction des flux des trains et des utilisateurs.

Figure 13.

Une vue datant des a $n$ n é e $s$ 1880 montre le Forest of Dean Tramroad, construit à partir de 1807 et toujours en service 80 a $n$ n é e $s$ plus tard,

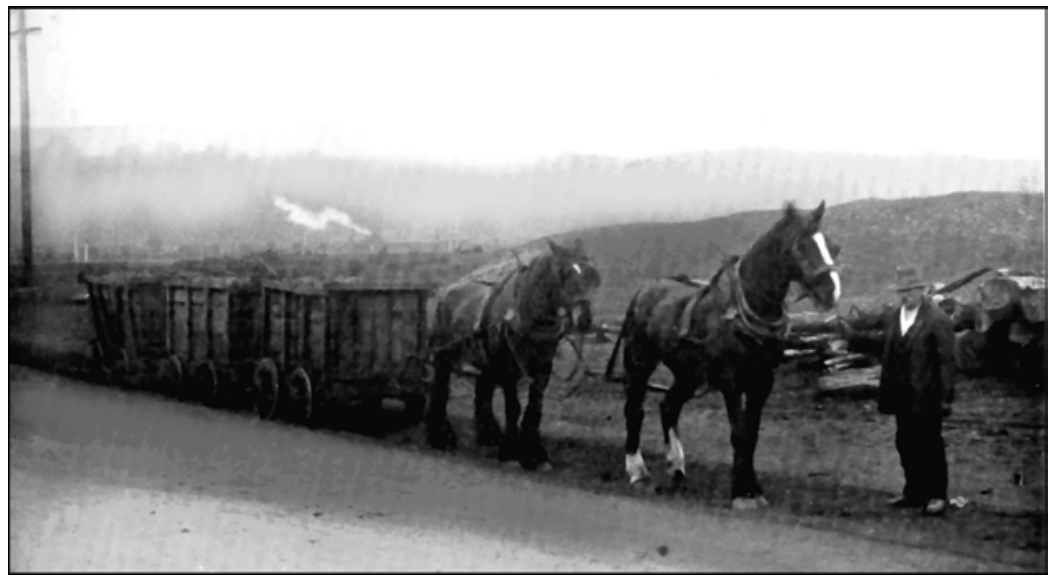

exactement dans sa forme primitive et avec le même type de matériel moteur (!) et roulant. Le réseau a atteint 180 miles, soit plus de 280 kilomètres lors de son extension maximale, et l'écartement est de 48 pouces, soit 1,22 mètre.

Figure 14. Forest of Dean Tramroad. Cette photographie, plus tardive que la précédente, montre, par les restes encore apparents, l'importance des installations ferroviaires et leur technicité. Des ouvrages d'art, des remblais et des tranchées ont été construits en grande quantité, préfigurant l'établissement des lignes de chemin de fer modernes. C'est, sans nul doute, le type de ligne que Seguin a pu visiter.

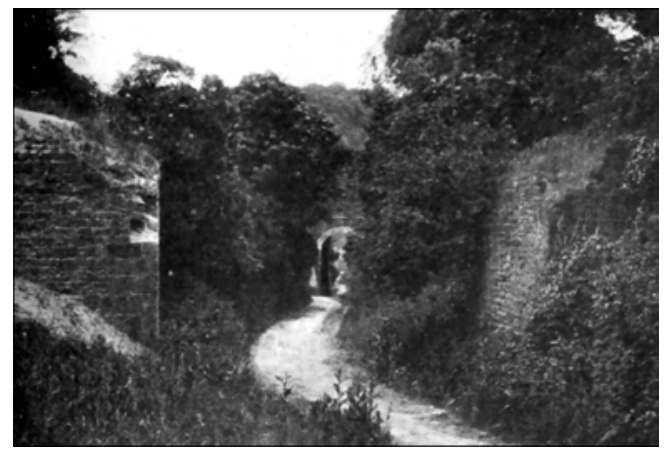




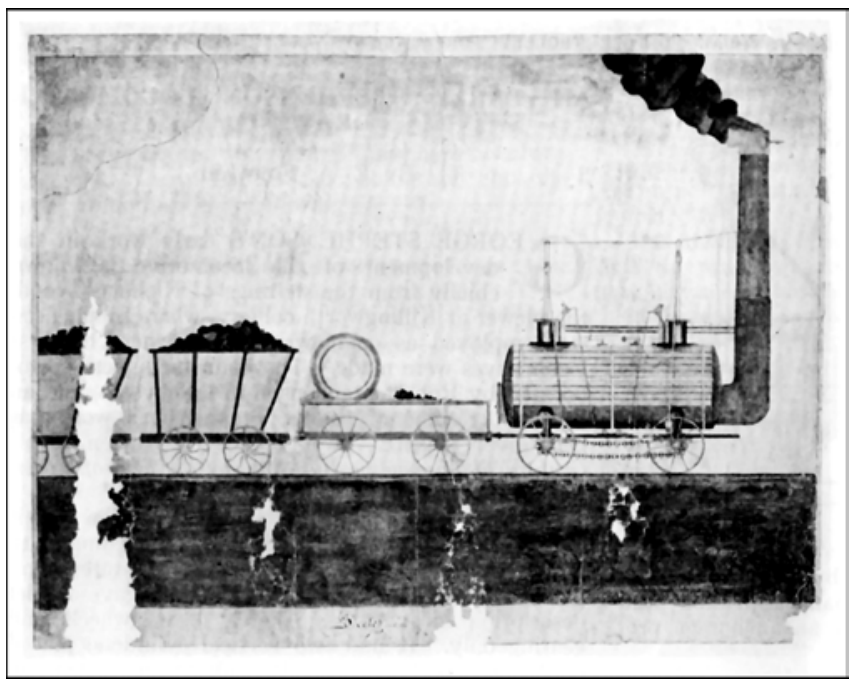

Figure 15. Une rare illustration de la locomotive conçue par Stephenson en 1815, sans doute la première, celle de Killingworth.

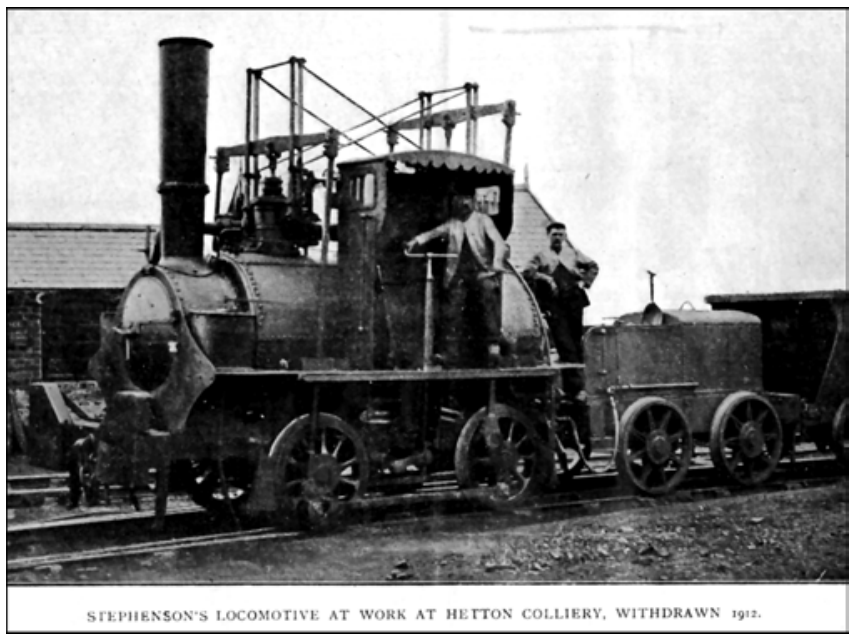

Figure 16. Une locomotive de Stephenson de 1822, photographiée peu avant son retrait en 1912. Cette vue exceptionnelle nous montre non seulement la longévité du matériel roulant mais l'influence de cette longévité sur la lente évolution technique du matériel due aux fortes contraintes d'écartement, de gabarit, d'attelage, etc. qui rendent difficile de s'en affranchir. 


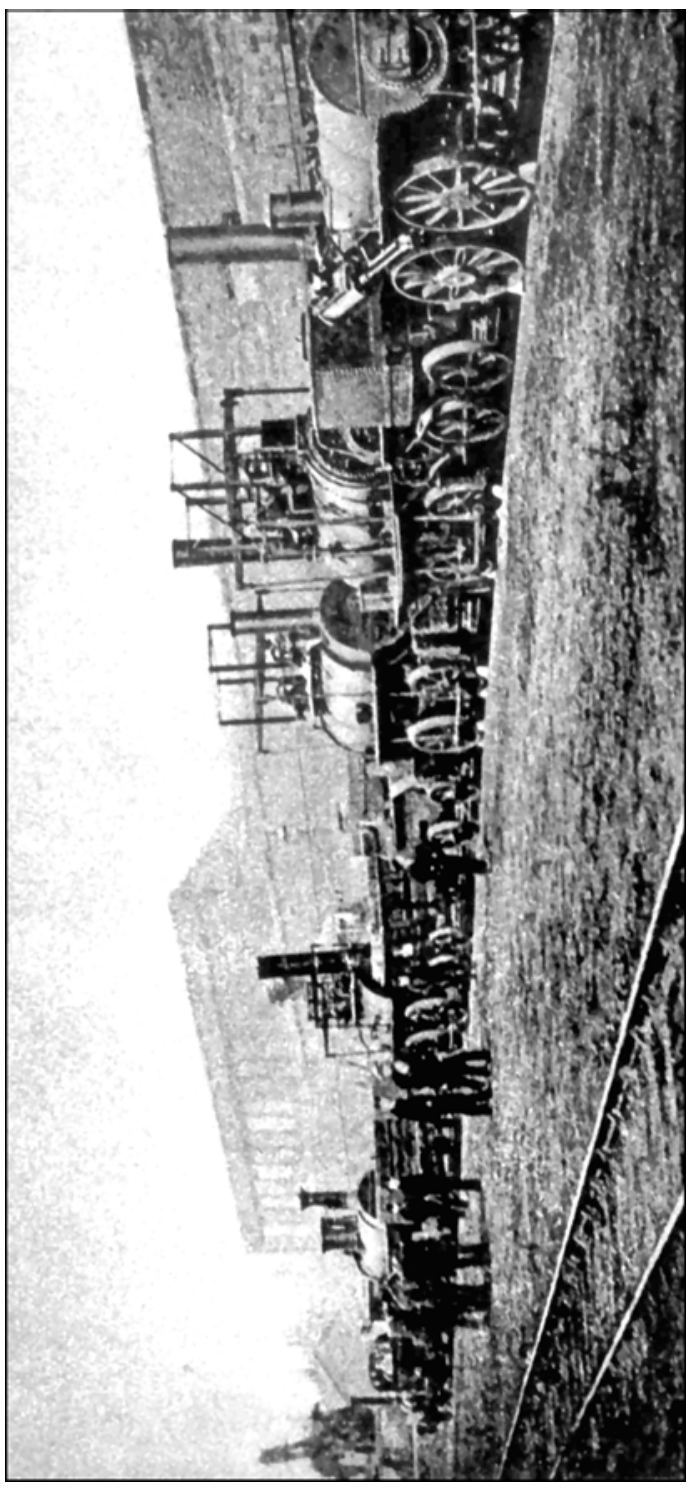

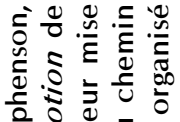

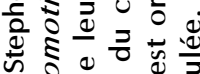

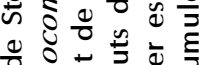

ช

过焉要

赵

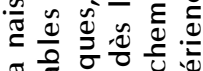

즈 $\frac{0}{2} \cdot \frac{\sigma}{5}$ 的

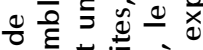

ญ 仓

कै थ

릴웡

屯

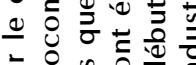

흐의 웡흥

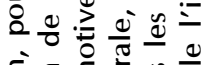

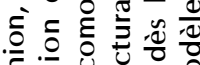

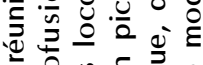

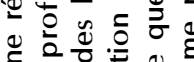

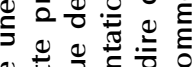

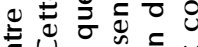

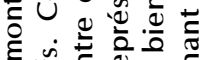

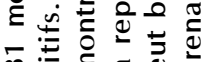

क

๘

$\approx$ in $\infty$ i

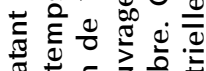

要

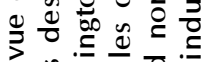
ป

人 $\stackrel{1}{\varepsilon}=\approx$

๙0

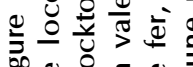

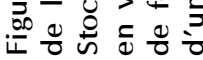


Visitant la ligne de Stockton à Darlington, il voit une ligne installée d'une manière très perfectionnée, avec un pont suspendu sur la rivière Tees dont la construction aura duré de 1824 à 1829, et dont le tablier est élargi en 1831 quand la ligne est mise à double voie. Il n'aura pas manqué de s'intéresser à ce gros pont suspendu, contemporain de ceux qu'il a jetés par-dessus le Rhône. Il aura contemplé la gare de Stockton, installée dans une maison ancienne qui devient un bâtiment ferroviaire le 23 mai 1822, et où l'on assure la gestion de la ligne, en attendant d'y vendre des billets à partir des années 1825 ou 1826.

\section{Ce que Marc Seguin sait}

Seguin sait beaucoup de choses : c'est un homme avisé, informé, qui a de nombreux correspondants. Il est au courant des brevets pris par Matthew Murray, l'un en 1801 concernant une distribution par soupapes à cames rotatives, l'autre en 1802 pour une distribution par tiroirs que lui achètera Trevithick pour ses essais de 1804 à Penydarran, dans le pays de Galles. Il sait, sans nul doute, que Woolf, un mécanicien des mines du pays de Galles, a déposé, en 1803, un brevet d'invention pour une chaudière tubulaire, et que la chaudière tubulaire de Woolf est utilisée à grande échelle dans les mines de Cornouailles pour l'alimentation des pompes à vapeur ${ }^{5}$. Il connaît la description de la locomotive de Blenkinshop qui roule en 1812 sur une voie à crémaillère latérale, capable de remorquer une charge de 94 tonnes à $8 \mathrm{~km} / \mathrm{h}$, et utilisant les brevets de Murray.

Marc Seguin a sans doute lu l'ouvrage de Nicholas Wood, Treatise on Railroads, publié en 1825, et qui semble être le premier ouvrage ferroviaire jamais paru. Dans ce livre, Wood décrit les locomotives de Stephenson, et tout particulièrement la première locomotive mise en chantier le 27 juillet 1814, comportant deux cylindres verticaux placés dans l'axe médian et à demi incorporés dans la chaudière. Seguin aura regardé l'illustration représentant une locomotive de type identique, datant de 1815, à roues accouplées par chaînes, illustration dessinée par Stephenson lui-même et que nous reproduisons ici (fig. 15).

5- Point crucial et objet de longues disputes entre historiens des techniques... Ni Seguin ni Stephenson n'ont inventé la chaudière tubulaire, et tous deux connaissent les travaux de Woolf dûment brevetés en 1803. Mais Seguin résout le problème de l'étanchéité de ce type de chaudière qui laissait fort à désirer à l'époque où il tente de l'appliquer sur ses locomotives. 
Ayant correspondu avec Stephenson, il connait déjà le chemin de fer anglais et les difficiles tentatives de la mise au point de la traction à vapeur. Il rentre avec la certitude de l'utilité des chemins de fer et la volonté de les transférer en France. Mais ce qu'il a pu voir dans de modestes bâtiments formant à la fois des ateliers et des dépôts l'a indubitablement conduit à une certaine méfiance vis-à-vis de la locomotive à vapeur.

\section{L'ère du " charronnage d'urgence "}

Déjà l'expérience de 1825, avec la Locomotion, est loin d'avoir été le triomphe que de nombreux peintres ou graveurs ont représenté, avec sa cheminée chauffée au rouge et un George Stephenson angoissé en train de la conduire. D'après des rapports d'époque écrits par des observateurs prussiens ${ }^{6}$, la traction à vapeur est loin d'avoir gagné tous les paris. Si cinq locomotives sont en service sur la ligne de Stockton à Darlington, et si elles sont certes capables de remorquer jusqu'à 24 wagons sur des rails secs, leur coût est très élevé si l'on tient compte des pannes, des accidents, des dégâts causés aux voies. La traction par chevaux coûte ainsi un tiers de la traction par locomotives à vapeur si l'on prend en compte tous les coûts indirects ${ }^{7}$. Les ateliers de la ligne sont submergés par des tâches innombrables regroupant à la fois ce qui, aujourd'hui, serait le fait des ateliers et des dépôts. Les premiers dépôts anglais puis français apparaissent plutôt comme des ateliers de dépannage d'urgence où de nombreux compagnons spécialisés s'affairent non seulement à réparer les locomotives mais aussi à les perfectionner pour les rendre fiables - créant sans nul doute une tradition qui se perpétuera dans les dépôts et les ateliers du monde entier, y compris en France où la maintenance a souvent sauvé du matériel moteur à l'origine défaillant.

La présence de charrons, de forgerons, de fondeurs, de chaudronniers, de menuisiers est le fait marquant. Le long des voies d'accès aux ateliers est stocké le charbon dans des stalles en bois ou par tas à même le sol, d'où il est chargé avec des paniers portés sur l'épaule par le chauffeur jusque dans les tenders. L'eau est refoulée par pompage dans des cuves surélevées à partir desquelles le chauffeur remplit les tonneaux placés en travers des tenders.

\footnotetext{
6- Von Deschen et Von Oeynhausen, «Observations on railways in England during the years 1826-1827 ", Archiv fur Bergbau und Hüttenwesen, Berlin, 1829.

7- Selon les termes mêmes du Report of the Committee of the Stockton \& $\sim$ Darlington Railway en date du 10 juillet 1827 .
} 
Dans les dépôts anglais, chaque mécanicien a la charge complète de sa machine, ceci conformément aux usages de la marine où le capitaine est en charge de son navire. Le mécanicien est rémunéré à la tonne remorquée et il paie son chauffeur. L'équipe James Stephenson et Robert Morrow, formant la première équipe de conduite régulière sur une ligne publique, touche $1 / 4$ de penny par tonne et par mille. Le mécanicien doit payer de sa poche le charbon, l'huile, le suif et le chanvre (pour les fuites), les fournitures diverses et tous les services du dépôt, accepter la remorque de wagons vides en retour. Les journées de conduite sont longues et se prolongent souvent tard le soir, le train étant signalé à l'arrière par un brasier de charbon incandescent. Les réparations au dépôt se font de nuit, les charrons, forgerons, chaudronniers, plombiers, menuisiers assurant un service à la demande du mécanicien et sous sa responsabilité ${ }^{8}$.

\section{La conception de l'espace dans les premiers dépôts français}

Plus que les gares, le dépôt est significatif de l'importation anglaise faite par Seguin. L'évolution se fait à partir du bâtiment rectangulaire primitif anglais, c'est à dire la remise - atelier rectangulaire avec accès par le pignon, dans l'axe du bâtiment. Le bâtiment évoluera par élargissement (multiplication des voies parallèles) et allongement (possibilité de garer plusieurs locomotives à la suite sur une voie). Cette disposition donne la grande remise rectangulaire avec faisceau d'accès se terminant par des voies parallèles.

Le dépôt britannique conservera longtemps cette disposition, et rares seront ceux pratiquant, pour le remisage des locomotives, le grand pont tournant avec sa rotonde. Il est à noter que les réseaux anglais pratiquent une curieuse forme : la rotonde «carrée » entourant le pont tournant, et comportant, en fin de compte, des voies de remisage de longueur inégale, celles aboutissant dans les angles du bâtiment étant plus longues...

En France, en revanche, le pont tournant et la rotonde apparaissent dès les années 1840 (gare de l'Est, remise de la Villette, par exemple) et, ignorées par Seguin, les rotondes seront la règle générale sur le réseau français jusqu'à ce que l' " EIMM »" actuel pour les TGV revienne en force à la disposition initiale à voies parallèles, comme c'est le cas particulièrement à Villeneuve-St-Georges ou au Landy...

8- Selon The Locomotive Magazine, numéro du 15 février 1928, p. 63.

9- Sigle de « l'établissement industriel de maintenance du matériel». Voir la communication d'A. Berheim dans le présent volume (N.d.l.R.). 


\section{La conception du travail dans les premiers dépôts français}

Important des locomotives anglaises, observateur du chemin de fer anglais, Marc Seguin est porté à adopter les méthodes de travail et d'organisation du chemin de fer d'outre-Manche. Lorsqu'il revient en France en 1827 pour construire la ligne de Saint-Étienne à Lyon, sa décision en faveur de la traction par locomotives n'est pas encore un fait acquis.

Les premières locomotives anglaises introduites en France, par Marc Seguin, sont similaires aux locomotives de la ligne de Bolton : des roues en bois cerclées de fer doux, cylindres verticaux entraînant les roues indirectement par un système de bielles et de renvois, absence de suspension. L'une des locomotives est livrée à Lyon, l'autre à Arras chez Halette pour transformation. Marc Seguin fait mention le 9 juin 1836 de deux locomotives anglaises « incapables de faire aucun service $»^{10}$ et exprime la même opinion dans son « État de situation au 30 avril $1835 »$ en faisant état d'un parc de 12 locomotives construites dans les ateliers de la compagnie et de deux locomotives anglaises qui n'ont jamais accompli de service satisfaisant. Il est intéressant de noter que les chevaux resteront en service sur la ligne jusqu'en 1844.

Marc Seguin, fort de ce qu'il a observé en Angleterre, se constitue des principes d'organisation du travail ${ }^{11}$ qui, aujourd'hui, prennent une valeur prémonitoire... si l'on considère qu'ils ont été écrits en 1838 :

- le refus d'«attirer des ouvriers étrangers en même temps que l'on déplace les industries » pour éviter d'avoir affaire à des gens motivés par le seul appât du gain, et des gens qui doivent fuir les lieux où ils ont donné une mauvaise impression (?).

- Ne pas laisser aux ouvriers la formation des apprentis, car « ces ouvriers sont extrêmement jaloux de leur savoir; ils souffrent avec peine les jeunes gens que l'on met auprès d'eux afin de les initier dans leur art et de les mettre en état de remplacer plus tard leurs maitres ». Ne pas laisser aux ouvriers la formation des apprentis parce que «les vices et les mauvaises habitudes qui sont la suite de toutes les grandes réunions d'ouvriers s'introduisent dans le nouvel atelier dès son origine, et y portent les germes de mécontentement et d'insubordination qu'il est ensuite si difficile de contenir $»$.

10- «Précis historique envoyé à Mr. Schmidt de St-Étienne ».

11- «De l'influence des chemins de fer... », op. cit., éd. 1887, p. 341. 
- Recruter et former soi-même, et à un haut niveau de compétence et de polyvalence : "Il vaut mieux, lorsqu'il y a possibilité de le faire, dresser des jeunes gens intelligents à conduire, réparer, et à construire les machines. »

Marc Seguin compte « peupler les ateliers de jeunes garçons chez lesquels se manifeste le goût de la mécanique, en les nourrissant de l'espérance de les préposer plus tard à la direction des machines qui se confectionnent sous leurs yeux». C'est ainsi que les ouvriers seront d'abord chauffeurs, puis passeront aux ateliers de montage des locomotives, les essaieront à la sortie de l'atelier, et les conduiront ensuite.

Seguin ne distingue donc pas, initialement, le dépôt des ateliers. Les machines sont entretenues et préparées dans des lieux servant aussi à la fabrication et à leur réglage, à leur perfectionnement. C'est encore une époque où l'on pratique un "chemin de fer de proximité ", ligne par ligne : les réseaux ne sont pas encore créés, mais, à partir de la fin des années 1830, ils vont se constituer et provoquer la création de grands ateliers et de nombreux dépôts séparés par de grandes distances et dispersés sur l'ensemble du territoire.

\section{Originalité et limites de l'apport de Marc Seguin}

Marc Seguin a plus marqué le chemin de fer français par sa conception de l'établissement des lignes que par les techniques ou les dépôts. Le point très fort de Seguin est d'avoir observé que les lignes anglaises de l'époque ont des tracés et des profils défavorables pour la pratique des vitesses élevées et pour le transport de lourdes charges. Homme de vision et d'avenir, Seguin sait que les trains iront de plus en plus vite et seront de plus en plus lourds. Il sait que la France est irréversiblement engagée dans une époque d'industrialisation intense, et que la demande de transport sera très forte.

C'est pourquoi il trace la ligne de Lyon à Saint-Étienne avec des courbes à grand rayon et des déclivités faibles, n’hésitant pas à déplacer des terres en grandes quantités, à construire des ouvrages d'art et des murs de soutènement multiples pour donner une plate-forme qui, aujourd'hui encore, est à la hauteur des exigences et accepte même la présence du TGV.

En revanche, Seguin n'a pas le souci - ou le temps - de prendre longuement et définitivement en charge une reconception du matériel moteur et remorqué. Si, en 1834, son parc moteur comprend dix locomotives Seguin assurant le transport de la houille (les voyageurs ont des trains remorqués par chevaux), en 1838, sur 15 locomotives, trois sont 
déjà des Planet anglaises et, peu à peu, les locomotives de conception Seguin sont éliminées pour laisser la place à des locomotives de conception Stephenson achetées en Angleterre ou construites en France. Seguin préfère, avec pragmatisme et lucidité, ne pas perdre son temps dans une technologie pour laquelle il ne se sent pas à la hauteur des fabricants professionnels anglais ou français et, peut-être à la suite de ce manque de succès technique, il quitte, en 1835, ses fonctions de directeur de la ligne de Lyon à Saint-Etienne. 